\title{
Article
}

\section{Nonlinear Viscoelastic Behavior of Aqueous Hybrid Threadlike Micellar Solutions}

\author{
Kenji NAKAMURA $^{* \dagger}$ and Toshiyuki SHIKATA \\ Department of Macromolecular Science, Osaka University, Toyonaka, Osaka 560-0043, Japan \\ (Received : February 6, 2008)
}

\begin{abstract}
Nonlinear viscoelastic behavior of aqueous hybrid threadlike micellar solutions consisting of cetyltrimethylammonium bromide (CTAB) and sodium poly(styrene-ran-sodium styrenesulfonate) (P(St/NaSS)) in water was investigated. Strain-hardening behavior well described with a three-(freely jointed)-chain model was observed when start-up shear flows at shear rates sufficiently higher than the reciprocal of the longest relaxation times were applied to the solutions. The size of segments, which is a reasonable measure of rigidity of hybrid threadlike micelles, evaluated from the number of segments necessary to make the best fit curves to shear stresses with the three-chain model were greater than that of the ordinary threadlike micelles consisting of CTAB and a low molar mass compound, sodium $p$-toluenesulfonate, possessing similar molecular structure to the monomer of $\mathrm{P}(\mathrm{St} / \mathrm{NaSS})$. Moreover, the molar mass of segments was independent of the molar mass of incorporated $\mathrm{P}(\mathrm{St} / \mathrm{NaSS}) \mathrm{s}$ in the hybrid threadlike micelles.
\end{abstract}

Key Words: Hybrid threadlike micelle / Supramolecular polymer / Polyelectrolyte-Surfactant complex /

Nonlinear viscoelasticity

\section{INTRODUCTION}

Threadlike micelles are constructed with addition of certain salts into an aqueous solutions of surfactants forming spherical micelles. ${ }^{1-3)}$ Shapes of threadlike micelles are thin flexible rods with uniform radii and long enough to entangle each other to show marked viscoelasticity. ${ }^{4,5)}$ Because threadlike micelles are formed by only intermolecular interactions between constituent molecules, they of course belong to the category of supramolecular polymers. Even at low surfactant concentrations less than $1 \mathrm{mM}$, enormously long threadlike micelles are formed between surfactants with long alkyl chains such as cetyltrimethylammonium bromide (CTAB) and hydrophobic aromatic additive salts like sodium salicylate $(\mathrm{NaSal})^{6)}$ or sodium $p$-toluenesulfonate (NapTS) ${ }^{7,8)}$ in aqueous solution (Scheme 1).

Viscoelastic behavior of aqueous fully entangled threadlike micellar systems shows unique rheological properties. The viscoelastic spectra of many threadlike micellar systems showing marked viscoelasticity are well described by a single Maxwell element with one set of relaxation time $(\tau)$

† E-mail: kenjin@se.ritsumei.ac.jp

present address: Department of Physical Sciences, Ritsumeikan University, Kusatsu, Shiga 525-8577, Japan and strength $\left(G_{\mathrm{N}}\right)$. Moreover, the viscoelastic behavior of aqueous threadlike micellar solutions is similar to that of concentrated polymer systems in some aspects. For example, the relationship $G_{\mathrm{N}} \propto c_{\mathrm{D}}^{2.0}$, where $c_{\mathrm{D}}$ means the concentration of surfactants, ${ }^{9)}$ is usually observed as well as in the polymer systems. In contrast, it has been well known that relaxation mechanisms of threadlike micellar systems are completely different from that of concentrated polymer systems in good solvent well described by, so called, a reptation mechanism. ${ }^{10)}$ The single Maxwell type viscoelastic behavior described above is of course one of characteristics of threadlike micellar systems which has been never observed in the polymer systems. In the concentrated polymer systems, the longest

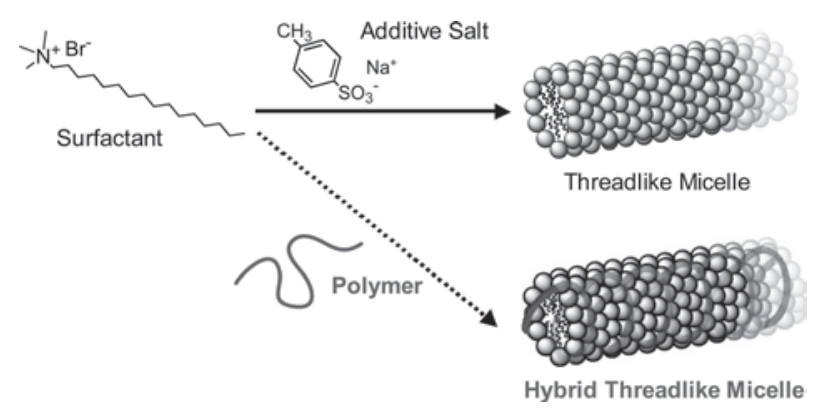

Scheme 1 
relaxation time is described as $\tau \propto M_{\mathrm{w}}{ }^{3.4} C_{\mathrm{P}}{ }^{1.6}$ with the molar mass $\left(M_{\mathrm{w}}\right)$ and concentration $\left(C_{\mathrm{P}}\right)$ of a polymer sample. ${ }^{10-12)}$ However, the value of $\tau$ for threadlike micellar systems do not depend on surfactant concentration, $c_{\mathrm{D}}$, but depends significantly on the concentration of additive salts.

Shikata et al. ${ }^{13)}$ proposed a phantom-crossing model to explain such the unique relaxation behavior observed in aqueous threadlike micelles. In the model, it is assumed that two entangled threadlike micelles form a temporary branching point at an entanglement point, and the entanglement is followed by a crossing-through reaction of the two micelles, as phantoms do when they contact or clash each other, after the lifetime of the entanglement point corresponding to $\tau$. The crossing-through reaction is accelerated by the exchange of additive salts between two states: the state of threadlike micelle forming with surfactant molecules on the micellar surface and that in bulk aqueous solution.

"Hybrid threadlike micelles" are classified into a group of threadlike micelles, and have definition that threadlike micelles consist of surfactant and polymer molecules instead of low molecular mass additive salts. ${ }^{14-21)}$ Then, we must define a term "ordinary threadlike micelles" to express threadlike micelles including only low molar mass molecules as additives. We recently have found that random copolymers of styrene and sodium styrenesulfonate $(\mathrm{P}(\mathrm{St} / \mathrm{NaSS}))$ forms stable hybrid threadlike micelles with CTAB in aqueous solution (Scheme 1). ${ }^{19)}$ Viscoelastic behavior of the hybrid threadlike micellar system, CTAB:P(St/NaSS)/W, was similar to those of fully entangled ordinary threadlike micellar systems $^{22)}$ and the relationship $G_{\mathrm{N}} \propto{C_{\mathrm{D}}}^{2.0}$ and $\tau \propto{C_{\mathrm{D}}}^{0}$ was recognized. The effect of incorporation of polymer chains in hybrid threadlike micelles was found as a change in a parameter, $\tau$, as follows. The value of $\tau$ for hybrid threadlike micellar solutions changed in proportion to the value of $M_{\mathrm{w}}$ of incorporated $\mathrm{P}(\mathrm{St} / \mathrm{NaSS})$ molecules.

In this study, we investigate the nonlinear viscoelastic behavior of hybrid threadlike micellar system, CTAB: $\mathrm{P}(\mathrm{St} / \mathrm{NaSS}) / \mathrm{W}$. It has been reported that ordinary threadlike micellar systems show nonlinear viscoelastic behavior much different from that of concentrated (entangled) polymer systems. ${ }^{9)}$ In the ordinary threadlike micellar systems, strain-hardening behavior was observed in their shear stress under stress relaxation measurements at large strains and also start-up shear flows at high shear rates, i.e., the initial shear modulus was higher than the linear value under large step strains, and the shear stress increased enormously and exhibited a marked overshoot much higher than the linear response at the onset of start-up shear flow. ${ }^{23-26)}$ Inoue et al. $^{25,26)}$ has reported that the significant strain-hardening behavior observed in an ordinary threadlike micellar system is attributed to the finite extensibility of threadlike micelles forming sticky temporal cross-links at entangling points which are not simple topological entanglements as those formed in the entangled polymer systems, but are temporal fused crosslinks as assumed in the phantom-crossing model. Since the molecular origin of strain-hardening behavior observed either in ordinary or hybrid threadlike micellar systems is directly related to characteristics of micellar structures, nonlinear viscoelastic measurements in the CTAB:P(St/NaSS)/W system provide useful information for the understanding of structure and dynamics of hybrid threadlike micelles.

\section{EXPERIMENTAL SECTION}

\subsection{Materials}

CTAB and NapTS were purchased from Wako Pure Chemicals Ltd. (Osaka) and were purified by recrystallization using methanol/acetone and water/methanol mixtures, respectively. Polystyrene (PSt) bearing number-average molecular weight, $M_{\mathrm{n}}$, $=27 \times 10^{3}$ with a measure of molecular weight distribution, $M_{\mathrm{w}} / M_{\mathrm{n}}$, $=1.17$, was purchased from Polymer Source Inc. (Montreal), and that bearing $M_{\mathrm{n}}=340 \times 10^{3}$ with $M_{\mathrm{w}} / M_{\mathrm{n}}=1.07$ was kindly supplied by TOSOH Corp. (Tokyo). Moreover, PSt bearing $M_{\mathrm{n}}=820 \times 10^{3}$ with $M_{\mathrm{w}} / M_{\mathrm{n}}=1.10$ was obtained by living anionic polymerization initiated by 2-methylbuthyllithium.

$\mathrm{P}(\mathrm{St} / \mathrm{NaSS}) \mathrm{s}$ were synthesized from PSts via a partial sulfonation technique by using acetylsulfonic acid proposed by Makowski. ${ }^{27)}$ The degree of sulfonation, $x_{\mathrm{S}}$, of $\mathrm{P}(\mathrm{St} / \mathrm{NaSS}) \mathrm{s}$ were determined using ${ }^{1} \mathrm{H}-\mathrm{NMR}$ measurements in DMSO- $d_{6}$ solution. $\mathrm{P}(\mathrm{St} / \mathrm{NaSS})$ s bearing $M_{\mathrm{n}}=27 \times 10^{3}$ with $x_{\mathrm{S}}=0.53$, $M_{\mathrm{n}}=340 \times 10^{3}$ with $x_{\mathrm{S}}=0.52$ and $M_{\mathrm{n}}=820 \times 10^{3}$ with $x_{\mathrm{S}}=0.53$ were used for sample preparation.

Highly deionized water with specific resistance higher than 15 M $\Omega \mathrm{cm}$ obtained using Milli-Q system (Japan Millipore, Tokyo) was used as a solvent for sample preparation.

CTAB:P(St/NaSS)/W with concentrations of total monomer units (styrene and sodium styrenesulfonate) of $\mathrm{P}(\mathrm{St} / \mathrm{NaSS})$, $c_{\mathrm{P}},=25 \mathrm{mM}$ and CTAB, $c_{\mathrm{D}},=25 \mathrm{mM}$ were prepared. The ordinary threadlike micellar system consisting of CTAB and NapTS with $c_{\mathrm{D}}=25 \mathrm{mM}$ and the concentration of NapTS, $c_{\mathrm{S}},=25 \mathrm{mM}$ (CTAB:NapTS/W) was also prepared.

\subsection{Measurements}

Linear and nonlinear viscoelastic measurements were performed using a stress-controlled rheometer, Physica MCR 
301 (Anton Paar, Graz), equipped with a measuring cell in the cone-and-plate geometry with the diameter of $50 \mathrm{~mm}$ and the cone angle of $2^{\circ}$. Under dynamic viscoelastic measurements, angular frequency $(\omega)$ was altered in a range from 0.003 to $100 \mathrm{~s}^{-1}$ and the amplitude of shear strain ranged in a low value region for the obtained storage and loss moduli ( $G^{\prime}$ and $G^{\prime \prime}$ ) enough to show the linear response. Shear rates $(\dot{\gamma})$ ranged from 0.0001 to $30 \mathrm{~s}^{-1}$ under start-up shear flow measurements. Magnitudes of shear strains $(\gamma)$ were altered from 0.1 to 8.0 under shear stress relaxation measurements. All the rheological measurements were carried out at $25^{\circ} \mathrm{C}$.

${ }^{1} \mathrm{H}-\mathrm{NMR}$ measurements for polymer sample characterization were performed using an EX-270 spectrometer (JEOL, Tokyo) in a deuterium-locked mode at $30^{\circ} \mathrm{C}$.

\section{RESULTS AND DISCUSSION}

\subsection{Linear Viscoelastic Behavior}

Before discussion of nonlinear viscoelastic behavior of the hybrid threadlike micellar system, CTAB:P(St/NaSS)/W, we briefly survey the linear viscoelastic behavior of the system. Fig. 1 shows the dependence of storage and loss moduli, $G^{\prime}$ and $G^{\prime \prime}$, on angular frequency, $\omega$, (viscoelastic spectrum) for CTAB:P(St/NaSS)/W containing polymers with different $M_{\mathrm{n}}$ at $x_{\mathrm{S}} \sim 0.50$ under the condition of $c_{\mathrm{D}}=c_{\mathrm{P}}=25 \mathrm{mM}$ and CTAB:NapTS/W at $c_{\mathrm{D}}=c_{\mathrm{S}}=25 \mathrm{mM}$. The longest relaxation times, $\tau$, evaluated from the reciprocals of the peak frequency in $G^{\prime \prime}$ curves for the CTAB:P(St/NaSS)/W solutions are always longer than that of the CTAB:NapTS/W system

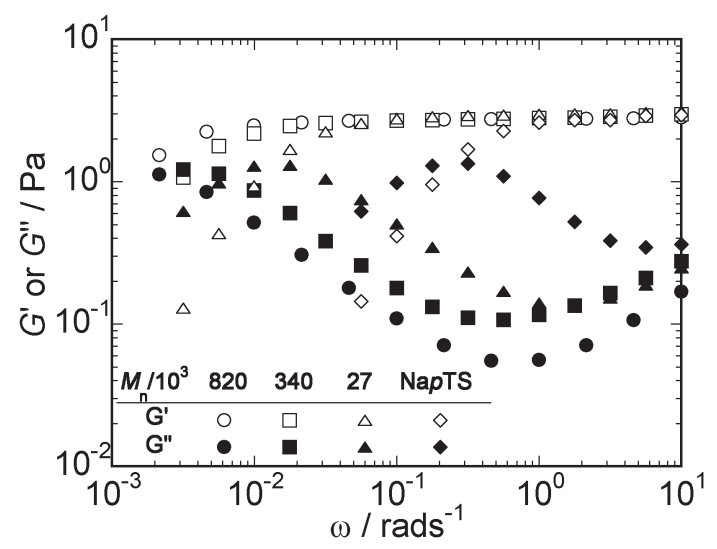

Fig. 1. Dependencies of storage and loss moduli, $G^{\prime}$ and $G^{\prime \prime}$, on angular frequency, $\omega$ for the CTAB:P(St/NaSS)/W system containing polymers with $M_{\mathrm{n}}=27 \times 10^{3}, 340 \times 10^{3}$ and $820 \times 10^{3}$ with about $x_{\mathrm{S}}=0.50$ under the condition of $c_{\mathrm{D}}=c_{\mathrm{P}}=25 \mathrm{mM}$ at $25^{\circ} \mathrm{C}$. The results for CTAB:NapTS/W with $c_{\mathrm{D}}=c_{\mathrm{S}}=25 \mathrm{mM}$ at $25^{\circ} \mathrm{C}$ are also shown in the figure. and become longer with increasing $M_{\mathrm{n}}$ of the incorporated polymers. Shapes of viscoelastic spectra ( $\omega$ dependence of $G^{\prime}$ and $\left.G^{\prime \prime}\right)$ for the CTAB:P(St/NaSS)/W system look very similar to each other irrespective of $M_{\mathrm{n}}$. However, the shapes of viscoelastic spectra for the hybrid threadlike micellar systems seems slightly broader than those observed in the ordinary threadlike micellar system, CTAB:NapTS/W. The values of $G_{\mathrm{N}}$ for both the CTAB:NapTS/W and CTAB:P(St/NaSS)/ W with different $M_{\mathrm{n}}$ are close to each other. These results well corresponding to those in the previous study ${ }^{22}$ indicate that incorporated polymers in hybrid threadlike micelles effectively interfere and retard the mechanical relaxation that is controlled by entanglement release mechanisms of hybrid threadlike micelles.

\subsection{Nonlinear Viscoelastic Behavior}

Fig. 2 shows viscosity growth functions $\left(\eta^{+}(t)\right)$ of the CTAB:P(St/NaSS)/W system containing a polymer with $M_{\mathrm{n}}=340 \times 10^{3}$ and $x_{\mathrm{S}}=0.53$ at $c_{\mathrm{D}}=c_{\mathrm{P}}=25 \mathrm{mM}$ under startup shear flow measurements at varying shear rates. The dotted line indicates a Maxwellian linear viscoelastic response $\left(\eta^{+}{ }_{\mathrm{L}}(t)\right)$ calculated from the following equation with the parameters of $G_{\mathrm{N}}=2.8 \mathrm{~Pa}$ and $\tau=280 \mathrm{~s}$ obtained from dynamic viscoelastic measurements shown in Fig. 1.

$$
\eta_{\mathrm{L}}^{+}(t)=G_{\mathrm{N}} \tau\left[1-\exp \left(-\frac{t}{\tau}\right)\right]
$$

The behavior of $\eta^{+}(t)$ curves seen in Fig. 2 can be categorized

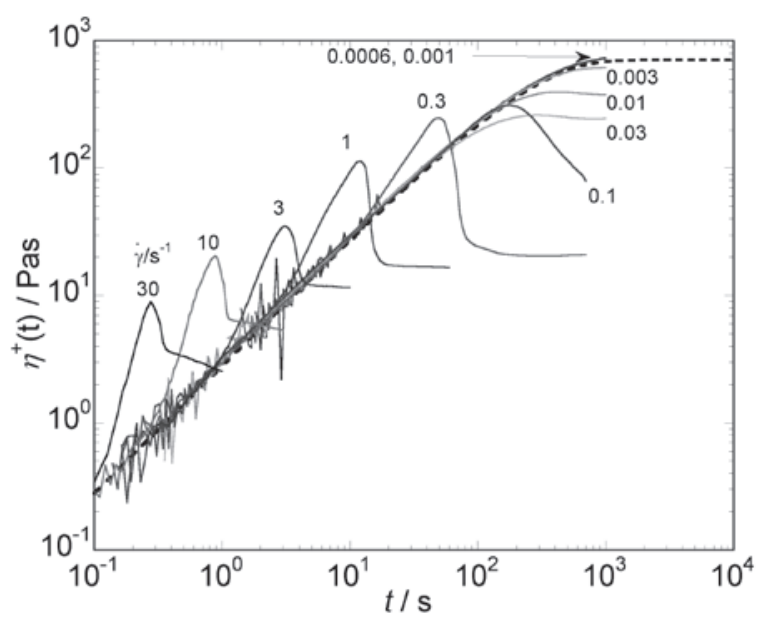

Fig. 2. Viscosity growth function, $\eta^{+}(t)$, for the CTAB:P(St/NaSS)/W system containing a polymer with $M_{\mathrm{n}}=340 \times 10^{3}$ and $x_{\mathrm{S}}=0.53$ under the condition of $c_{\mathrm{D}}=c_{\mathrm{P}}=25 \mathrm{mM}$ at $25{ }^{\circ} \mathrm{C}$. A dotted line indicates the Maxwell-type behavior, $\eta_{\mathrm{L}}^{+}(t)$, in the linear viscoelastic limit calculated from Eq. (1). 
into three types. The first type is "linear behavior" showing the relationship $\eta^{+}(t)=\eta_{\mathrm{L}}^{+}(t)$, observed at a $\dot{\gamma}$ range lower than $0.001 \mathrm{~s}^{-1}$. The second type shows "shear thinning behavior", in which the relationship $\eta^{+}(t) \leq \eta_{\mathrm{L}}^{+}(t)$ observed in the steady state (at $t$ much longer than $\tau$ ) at a $\dot{\gamma}$ range higher than $0.003 \mathrm{~s}^{-1}\left(=\tau^{-1}\right)$. In this type, $\eta^{+}(t)$ shows a weak overshoot, and then reaches the steady state value holding the relationship $\eta^{+}(t) \leq \eta_{\mathrm{L}}^{+}(t)$. The third type shows clear, marked overshoot much higher than the linear response, which means "strain-hardening behavior". The relationship $\eta^{+}(t)>\eta_{\mathrm{L}}^{+}(t)$ is observed at $\dot{\gamma}>0.3 \mathrm{~s}^{-1}$ in the stress overshoot regime. In the third type, $\eta^{+}(t)$ suddenly increases and deviates upward from the $\eta_{\mathrm{L}}^{+}(t)$ curve, and is followed by a stress-overshoot peak, and then drastically decreases to a constant value much lower than $\eta_{\mathrm{L}}^{+}(t)$ curve in the steady state.

The strain-hardening behavior was also observed under stress relaxation measurements at large step strains. Fig. 3 shows the results of the stress relaxation measurements for the same CTAB:P(St/NaSS)/W system as shown in Fig. 2. The relaxation modulus $(G(t, \gamma))$ is independent of shear strain, $\gamma$, and coincides with the relationship $G_{\mathrm{N}} \mathrm{e}^{-t / \tau}$ calculated from the linear responses when the applied $\gamma$ value was less than 2. However, the magnitude of $G(t, \gamma)$ at $t=0.1 \mathrm{~s}\left(G_{0}\right)$, which is close to the instantaneous value at $t=0$, increased with increasing $\gamma$ greater than 3 . In this regime, the system instantaneously hardened at the onset of large strains. At $\gamma$ higher than 8, $G_{0}$ decreased probably due to slippage of samples on the surface of measuring cone-and-plate cell and/ or the fracture of hybrid threadlike micelles caused by large deformations due to high $\gamma$ values. The time dependence of $G(t, \gamma)$ in a long time region after the initial stress-hardening behavior is well described with a single relaxation time

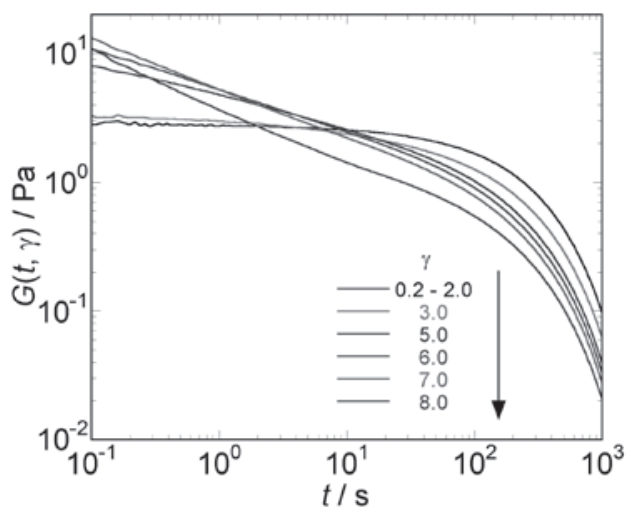

Fig. 3. Stress relaxation modulus, $G(t, \gamma)$, for the CTAB:P(St/NaSS)/W system containing a polymer with $M_{\mathrm{n}}=340 \times 10^{3}$ and $x_{\mathrm{S}}=0.53$ at $c_{\mathrm{D}}=c_{\mathrm{P}}=25 \mathrm{mM}$ and $25^{\circ} \mathrm{C}$. identical with $\tau$ determined in the linear regime, while the amplitude of the relaxation is dramatically reduced with increasing $\gamma$. This behavior observed in the long time region looks like the time dependence of $G(t, \gamma)$ for entangled polymer systems also in the long time region. It has been well known that entangled polymer systems show two-step relaxation behavior under stress relaxation experiments at large strains: a short time relaxation related to a contraction mode of extended polymer chains and a long time relaxation related to a reptation mode for entanglement release. ${ }^{10)}$ However, the entangled polymer system never show strainhardening behavior in the contraction mode with the short relaxation time in contrast with the findings in the hybrid threadlike micellar systems seen in Fig. 3.

Similar nonlinear viscoelastic behavior seen in Fig. 2 and Fig. 3 were observed in other CTAB:P(St/NaSS)/W systems containing polymers with $M_{\mathrm{n}}=27 \times 10^{3}$ and $x_{\mathrm{S}}=0.53$, and $M_{\mathrm{n}}=820 \times 10^{3}$ and $x_{\mathrm{S}}=0.53$ at the same micellar concentration. As stated in the introductory part, similar nonlinear behavior was also observed in ordinary threadlike micellar systems consisting of CTAB and NaSal (CTAB: $\mathrm{NaSal} / \mathrm{W})^{23-26)}$ and CTAB:NapTS/W.

The effective shear modulus $\left(G_{\text {eff }}\right)$ can be evaluated from $\eta^{+}(t)$ in the manner given by Eq. (2), ${ }^{25)}$ and corresponds to the instantaneous shear modulus when the applied $\dot{\gamma}$ is much higher than the reciprocal of the longest relaxation time of an examined system.

$$
G_{\text {eff }}=\frac{\eta^{+}(t)}{t}
$$

Fig. 4(a) shows the dependence of $G_{\text {eff }}$ on macroscopic strain, $\gamma(=\dot{\gamma} t)$, for the same system as shown in Fig. 2. $G_{\text {eff }}$ curves increased with $\gamma$ and were followed by maximums at $\dot{\gamma}>0.3 \mathrm{~s}^{-1}$. With increasing $\dot{\gamma}$, the initial upturns of $G_{\text {eff }}$ curves at $\gamma \sim 3$ converge on the limiting behavior obtained at high $\dot{\gamma}$. The clear agreement between the limiting initial $G_{\text {eff }}$ curve up to $\gamma=5$ obtained at $\dot{\gamma} \geq 6 \mathrm{~s}^{-1}$ and the $G_{0}$ values (large opened circle) obtained from the stress relaxation measurements supports the reliability of the results obtained from these different nonlinear viscoelastic techniques each other.

\subsection{Segment Size of Hybrid Threadlike Micelles}

Inoue et al. ${ }^{25)}$ have analyzed the strain-hardening behavior of the ordinary threadlike micellar system of CTAB:NaSal/W using a three-(freely jointed)-chain model. ${ }^{28,29)}$ In this model, threadlike micelles were regarded as non-gaussian freely jointed chains with a finite segment number giving finite 
extensibility. This model is based on the assumption that a cubic network may be replaced by three independent sets of freely jointed chains, of which terminals are pined on the central points of each facing side of the network cube. The three-chain model provides $\gamma$ dependent shear modulus $(G(\gamma))$ as given by Eq. (3) due to the characteristics of freely joined chains. $^{28,29)}$

$$
G(\gamma)=\frac{G_{N} n^{1 / 2} \sin \chi \cos \chi}{3 \gamma}\left[\lambda L^{-1}\left(\frac{\lambda}{n^{1 / 2}}\right)-\frac{1}{\lambda} L^{-1}\left(\frac{1}{\lambda n^{1 / 2}}\right)\right]
$$

where

$$
\lambda=\frac{\gamma+\sqrt{\gamma^{2}+4}}{2} \text { and } \chi=\cot ^{-1} \lambda
$$

The parameters, $n$ and $\lambda$, are defined as the number of segments per constituent freely jointed chain and stretch ratio of the chain under the shear deformation at $\gamma$, respectively. $L^{-1}(x)$ means an inverse Langevin function of $x$. The parameter, $n$, in Eq. (3) only determines $\gamma$ dependence of $G(\gamma)$. We employ a Padé approximation of the inverse Langevin function as $L^{-1}(x)$ for curve fit procedures of $G(\gamma)$ to experimental $G_{\text {eff }}$ and $G_{0}$ curves. $^{30}$

A thick solid curve in Fig. 4(a) represents theoretical $G(\gamma)$ calculated via Eq. (3) with the parameter of $n=29$. The curve agrees well with the $G_{\text {eff }}$ curves at high $\dot{\gamma}$ and also with the $G_{0}$ curves. The $G_{\text {eff }}$ curves for the CTAB:P(St/NaSS)/W system with different $M_{\mathrm{n}}$ were also well fitted using Eq. (3).
Fig. 4(b) shows the relationship between $G_{\text {eff }}$ and $G_{0}$, and $G(\gamma)$ obtained in the ordinary threadlike system, CTAB:NapTS/ $\mathrm{W}$ at the same micellar concentration. Essentially the same behavior as seen in Fig. 4(a) is observed other than the large $n$ value of 140 . The obtained $n$ values for the hybrid threadlike micellar system and the ordinary threadlike micellar system of CTAB:NapTS/W with the same concentration at $25 \mathrm{mM}$ are summarized in Table I. The $n$ values for the CTAB:P(St/ $\mathrm{NaSS}$ )/W system with different $M_{\mathrm{n}}$ values are similar to each other and much smaller than that for the CTAB:NapTS/W system.

The molar mass of a network strand chain between entanglements $\left(M_{\mathrm{e}}\right)$ is calculated from the $G_{\mathrm{N}}$ data using the relationship

$$
M_{e}=\frac{c R T}{G_{N}}
$$

Table I. The number of segments per hybrid threadlike chain strand between entanglements: $n$, molar mass of hybrid threadlike chain strand between entanglements: $M_{\mathrm{e}}$, molar mass of the segment: $M_{\mathrm{s}}$, the number of the monomer units per segment: $N_{\text {seg }}$, for CTAB:NapTS/W and CTAB:P(St/NaSS)/W with various $M_{\mathrm{n}}$ and $x_{\mathrm{S}}$ of about 0.50 under the condition of equimolar concentrations of CTAB and NapTS or P(St/NaSS) of nearly $25 \mathrm{mM}$ at $25^{\circ} \mathrm{C}$.

\begin{tabular}{cccccc}
\hline sample & $M_{\mathrm{n}}$ & $n$ & $M_{\mathrm{e}} / 10^{6}$ & $M_{\mathrm{S}} / 10^{4}$ & $N_{\text {seg }}$ \\
\hline CTAB:NapTS & ------ & 140 & 9.9 & 7.1 & 155 \\
CTAB:P(St/NaSS) & $27 \times 10^{3}$ & 24 & 8.7 & 36 & 834 \\
CTAB:P(St/NaSS) & $340 \times 10^{3}$ & 29 & 10 & 35 & 810 \\
CTAB:P(St/NaSS) & $820 \times 10^{3}$ & 22 & 9.1 & 41 & 915 \\
\hline
\end{tabular}

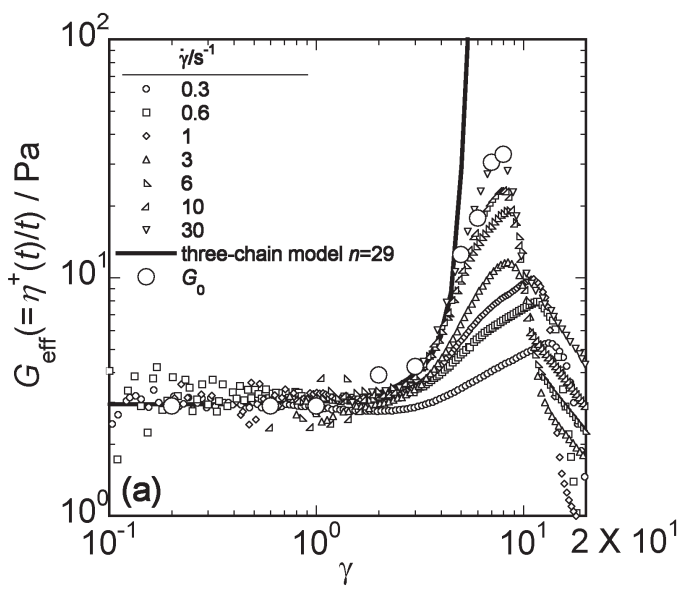

(a)

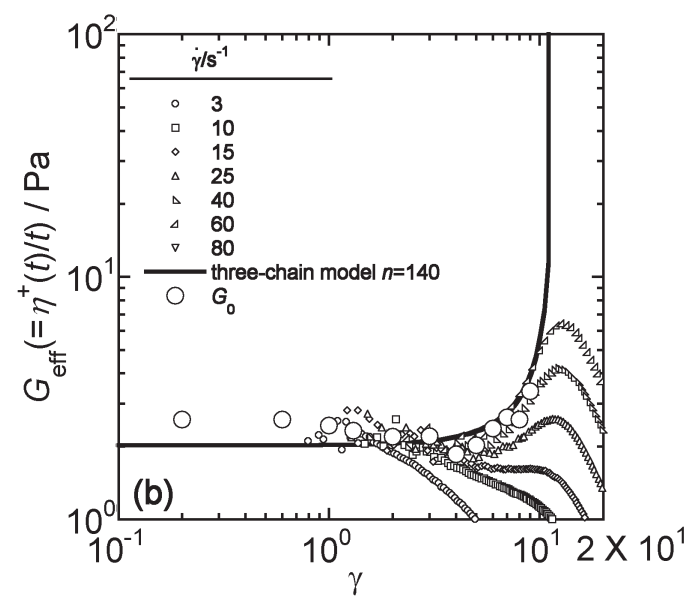

(b)

Fig. 4. (a): Strain, $\gamma$, dependence of effective modulus, $G_{\text {eff }}$, for CTAB:P(St $\left./ \mathrm{NaSS}\right) / \mathrm{W}$ system containing a polymer with $M_{\mathrm{n}}=340 \times 10^{3}$ and $x_{\mathrm{S}}=0.53$ at $c_{\mathrm{D}}=C_{\mathrm{p}}=25 \mathrm{mM}$ and $25{ }^{\circ} \mathrm{C}$. Strain dependence of $G_{0}$ values obtained from stress relaxation measurement for the same system are also shown in the figure (large opened circle). A solid line represents a theoretical $G(\gamma)$ curve calculated by three-(freely jointed)-chain model (Eq. (3)) with the parameter of $n=29$. (b): The dependence of $G_{\text {eff }}$ and $G_{0}$ on $\gamma$ for the CTAB:NapTS/W system. A solid line represents a theoretical $G(\gamma)$ curve calculated by three-(freely jointed)-chain model with the parameter of $n=140$. 
where $c$ is the mass concentration of a monomer unit. In the case of the CTAB:P(St/NaSS)/W system, the molar mass of a monomer unit $\left(M_{0}\right)$ should be evaluated to be the average molar mass of a 1:1 polymer-surfactant complex unit consisting of a $\mathrm{CTA}^{+}$cation and $\mathrm{SS}^{-}$or St units in $\mathrm{P}\left(\mathrm{St} / \mathrm{SS}^{-}\right)$ chains, moreover, in the CTAB:NapTS/W system the molar mass of a 1:1 complex between $\mathrm{CTA}^{+}$and $p \mathrm{TS}^{-}$. Because the molar mass of the segment $\left(M_{\mathrm{s}}\right)$ is defined as $M_{\mathrm{s}}=M_{\mathrm{e}} / n$, the number of monomer units per segment $\left(N_{\text {seg }}\right)$ is calculated as $N_{\text {seg }}=M_{\mathrm{s}} / M_{0}$. The evaluated $M_{\mathrm{e}}, M_{\mathrm{s}}$, and $N_{\text {seg }}$ values for the CTAB:P(St/NaSS)/W and CTAB:NapTS/W systems are also tabulated in Table I. Comparing the $N_{\text {seg }}$ value for the CTAB:NapTS/W system with those of the CTAB:P(St/ $\mathrm{NaSS}$ )/W system leads to two significant conclusions; the $N_{\text {seg }}$ value of the CTAB:P(St/NaSS)/W system is more than five times as large as that of the CTAB:NapTS/W system, and is approximately independent of the $M_{\mathrm{n}}$ values of incorporated polymers.

Since the $N_{\text {seg }}$ value is a reasonable measure of the rigidity of threadlike micelles, the former conclusion indicates that hybrid threadlike micelles are more rigid than the ordinary threadlike micelles and the latter implies that the rigidity of the hybrid threadlike micelle is independent of $M_{\mathrm{n}}$ of incorporated polymers. One would accept the former conclusion naturally because the presence of incorporated polymers with sequential chemical bonds in threadlike micelles possibly stiffens the micelles more than that of small additive, $p \mathrm{TS}^{-}$. The latter conclusion is acceptable for the solutions with polymers of $M_{\mathrm{n}} \geq 340 \times 10^{3}$ since their average degrees of polymerization (the numbers of the $1: 1$ complex units in a polymer) are a few times as large as $N_{\text {seg}}$, which means that the length of hybrid threadlike micelles formed by polymers is a few times longer than that of the segment. Such a kind of discussion on the length of hybrid threadlike micelles makes sense, because small angle neutron scattering (SANS) experiments in the same hybrid threadlike micellar system ${ }^{31)}$ as used this study revealed that the average number of polymer chains on the crosssection of the hybrid threadlike micelle is 1.3 irrespective of $M_{\mathrm{n}}$, and the incorporated polymers in the hybrid threadlike micelle are partially overlap each other. The fact that the $N_{\text {seg }}$ value for the solution containing a polymer with the smallest $M_{\mathrm{n}}=27 \times 10^{3}$ is almost the same as those for other solutions suggests that the presence of junction portions for incorporated polymers in the hybrid threadlike micelle does not affect the rigidity of the micelle. Consequently, the length of incorporated polymer in the micelle may less affect the rigidity of the micelles.

\subsection{Relaxation Mechanism for Hybrid Threadlike Micellar System}

In general, the entangled linear polymer systems merely show strain-hardening behavior even under strong shear deformation like large step strains or start-up flows. ${ }^{9)}$ The contraction process of polymer chains with finite lengths is a major molecular mode related to viscoelasticity in a time scale shorter than the longest relaxation time, $\tau$, which prevents the polymer chains from the highly stretched state due to their finite extensibility. Thus, the shear thinning behavior is usually observed in entangled polymer systems. According to Inoue et al., ${ }^{25,26)}$ the strain-hardening behavior observed in the threadlike micellar system, CTAB:P(St/NaSS)/W, is attributed to the finite extensibility of the hybrid threadlike micelles forming fused temporal cross-links at entanglements as assumed in the phantom-crossing model. ${ }^{13)}$ Consequently, the relaxation mechanism for the hybrid threadlike micellar system is essentially explained with the phantom-crossing model as proposed in the previous study. ${ }^{22)}$

A living polymer model proposed by Cates is another basic, important relaxation model for the viscoelasticity of the ordinary threadlike micellar system. ${ }^{32,33)}$ In this model, it is assumed that threadlike micelles reptate like polymer chains with finite lengths in entangled networks of themselves, and repeat scission at any places on their long strands and reformation at contacting two terminals. The living polymer model always predicts increment of $\tau$ with increasing the concentration of threadlike micelles in the linear viscoelastic regime. Since $\tau$ of CTAB:P(St/NaSS)/W were independent of the concentration of CTAB, $c_{\mathrm{D}}$, we already have concluded that the living polymer model can not explain the relaxation mechanism of hybrid threadlike micelles. ${ }^{22)}$ The living polymer model also predicts the shear thinning behavior under non-linear viscoelastic regime as in the case of the concentrated polymer system because threadlike micelles assumed in the living polymer model possess two terminals proving the finite micellar length and the contraction mode in a short time scale. ${ }^{33,34)}$ Disagreement between the nonlinear viscoelastic behavior for the CTAB: $\mathrm{P}(\mathrm{St} / \mathrm{NaSS}) / \mathrm{W}$ system (strain-hardening behavior) and prediction by the living polymer model (shear thinning behavior) reveals that the relaxation mechanism of hybrid threadlike micelles are not explained by the living polymer model, but the phantom-crossing model as in the ordinary threadlike micellar systems. 


\section{CONCLUSION}

We investigate nonlinear viscoelastic behavior of hybrid threadlike micelles consisting of cetyltrimethylammonium bromide and poly(styrene-ran-sodium styrenesulfonate) in aqueous solution. This system shows significant strainhardening behavior under start-up flows at high shear rates like ordinary threadlike micellar systems consisting of surfactant and low molar mass additives. This strain-hardening behavior is well explained by the three-(freely jointed)-chain model. Segment size of the hybrid threadlike micelle is longer than that of the ordinary threadlike micelle and is kept constant irrespective of the molar mass of incorporated polymers into the hybrid threadlike micelle. The strain-hardening behavior observed strongly suggests that the relaxation mechanism of the hybrid threadlike micellar system is governed by the phantom-crossing process at entanglement points between two entangled hybrid threadlike micelles.

\section{Acknowledgement}

K.N. wishes to express special thanks for "Research Fellowship for Young Scientists” from the Japan Society for the Promotion of Science.

\section{REFERENCES}

1) Tanford C, "The Hydrophobic Effect: Formation of Micelles and Biological Membranes”, $2^{\text {nd }}$ ed. (1980), Wiley, New York.

2) Israelachivili JN, “Intermolecular \& Surface Forces”, $2^{\text {nd }}$ ed. (1991), Academic Press, New York.

3) Magid LJ, J Phys Chem B, 102, 4064 (1998).

4) Rehage H, Hoffmann H, Mol Phys, 74, 933 (1991).

5) Rehage H, Hoffmann H, J Phys Chem, 92, 4712 (1988).

6) Shikata T, Hirata H, Kotaka T, Langmuir, 3, 1081 (1987).

7) Soltero JFA, Puig JE, Langmuir, 12, 2654 (1996).

8) Shikata T, Shiokawa M, Imai S, J Colloid Interface Sci, 259, 367 (2003).

9) Ferry JD, "Viscoelastic Properties of Polymers", $3^{\text {rd }}$ ed. (1980), John Wiley, New York.
10) Doi M, Edwards SF, “The Theory of Polymer Dynamics”, $3^{\text {rd }}$ ed. (1980), John Wiley, New York.

11) de Gennes P-G, "Scaling Concepts in Polymer Physics", (1979), Cornel University Press, Ithaca, New York.

12) Rubinstein M, Colby RH, “Polymer Physics”, (2003), Oxford University Press, London.

13) Shikata T, Hirata H, Kotaka T, Langmuir, 4, 354 (1988).

14) Kline SR, Langmuir, 15, 2726 (1999).

15) Wu H, Kawaguchi S, Ito K, Colloid Polym Sci, 282, 1365 (2004).

16) Liu S, González YI, Damino D, Kaler EW, Macromolecules, 38, 2482 (2005).

17) Zhu Z, González YI, Hangxun X, Kaler EW, Liu S, Langmuir, 22, 949 (2006).

18) Nakamura K, Shikata T, ChemPhysChem, 8, 2568 (2007).

19) Nakamura K, Shikata T, Macromolecules, 36, 9698 (2003).

20) Nakamura K, Shikata T, J Phys Chem B, 110, 24802 (2006).

21) Nakamura K, Yamanaka K, Shikata T, Langmuir, 19, 8654 (2003).

22) Nakamura K, Shikata T, Macromolecules, 37, 8381 (2004).

23) Shikata T, Hirata H, Takatori E, Osaki K, J Non-Newtonian Fluid Mech, 28, 171 (1988).

24) Brown EF, Burghardt WR, Venerus DC, Langmuir, 13, 3902 (1997).

25) Inoue T, Inoue $Y$, Watanabe $H$, Langmuir, 21, 1201 (2005).

26) Takagi A, Sasaki H, Kuriyama A, Matsumiya $Y$, Inoue $T$, Watanabe H, Nihon Reoroji Gakkaishi, 34, 15 (2006).

27) Makowski HS, Lundberg RD, Singhal GH, US Patent 3,870,841. 1975.

28) Wang MC, Guth E, J Chem Phys, 20, 1144 (1952).

29) Treloar LRG, "The Physcis of Rubber Elasticity", (1958), Clarendon, Oxford, UK.

30) Cohen A, Rheol Acta, 30, 270 (1991).

31) Nakamura K, Shikata T, J Phys Chem B, 111, 12411 (2007).

32) Cates ME, Macromolecules, 20, 2289 (1987).

33) Cates ME, Candau SJ, J Phys: Condens Matter, 2, 6869 (1990).

34) Cates ME, J Phys Chem, 94, 371 (1990). 